\title{
Interleukin-22 receptor is overexpressed in nonsmall cell lung cancer and portends a poor prognosis
}

To the Editor:

The recent decade has witnessed a change of view about cancer mechanisms. Considerable evidence now suggests that the tumour microenvironment status is well correlated with disease outcome. The presence of particular immune cell types or molecules determines whether a pro- or antitumour immune response predominates within the microenvironment [1]. Accordingly, improved understanding of the factors that modulate the tumour microenvironment will be critical for the development of effective future strategies for cancer management.

In this regard, interleukin (IL)-22, a member of the IL-10 family, has attracted considerable attention as it is closely related to the initiation and development of cancer [2]. IL-22 is produced by innate lymphoid cells, mediates its cellular effects via a heterodimer receptor complex composed of two different subunits (IL-22 receptor subunit 1 (IL-22R1) and IL-10R2), and signals through signal transducer and activator of transcription (STAT) molecules [3]. The IL-10R2 subunit is ubiquitously expressed. IL-22R1 determines cellular sensitivity towards IL-22 and is exclusively expressed by the epithelial cells [4]. Thus, IL-22 is unusual among interleukins because it does not directly regulate the function of archetypical immune cells, i.e. leukocytes, but instead targets cells at outer body barriers, such as respiratory epithelial cells, and induces expression of genes involved in tissue homeostasis [3].

Remarkably, IL-22 signalling is a critical orchestrator of pro-survival, cell migration, mitogenic and anti-apoptotic effects and these functions can be hijacked by aggressive cancers to enhance tumour growth and metastasis [2]. Also, functional studies in murine model systems indicate that IL-22/IL-22R has immunomodulatory properties, including in cancer [2]. IL-22 expression was shown to correlate positively with oncogenesis and staging of tumours of the gastrointestinal tract as well as hepatocellular carcinomas [2]. IL-22 has also been shown to improve the invasive ability of pancreatic cancer cell lines by increasing matrix metalloprotease 9 [2]. It is of note, however, that there have been limited studies on IL-22/IL-22R alterations in lung cancers [5-7]. In 2008, ZHANG et al. [7] reported that IL-22 gene expression was enhanced in tumour tissues of six patients with a histological diagnosis of primary nonsmall cell lung cancer (NSCLC). KoBold et al. [5] confirmed such IL-22 dysregulation in NSCLC but could not correlate this overexpression with occurrence and progress of NSCLC, despite a study on a larger patient cohort. Regardless, we believe that when one considers lung cancer, it is more pertinent to focus on the regulation of IL-22R per se rather than the cytokine IL-22, as IL-22R-bearing epithelial cells are likely to be more immediately exposed to harmful airborne contaminants, including tobacco-related carcinogens, than the innate lymphoid cells secreting IL-22. Importantly, we and others previously demonstrated that IL-22R1 subunit expression is regulated (positively or negatively) at lung barrier surfaces where epithelial cells play an active role in the initiation, regulation and resolution of immune responses [8-10].

In view of this, our study aimed first to investigate IL-22R1 dysregulation in a large cohort of lung cancer patients and, secondly and more importantly, to assess its association with overall survival.

We collected lung tissues from patients who underwent surgery for NSCLC between 2006 and 2011 (application number for declaration to Ministère de l'Enseignement Supérieur et de la Recherche: DC-2008-308). Informed written consent was obtained from each participant. To determine whether IL-22R1 subunit expression was regulated at the level of gene transcription, total RNA was isolated from homogenates of tumours as well as from histologically noncancerous lung tissues adjacent to the tumour $(\mathrm{n}=81)$. All samples were processed for quantitative reverse transcriptase PCR. As shown in figure 1a, IL-22R1 gene expression was more than two-fold higher in lung cancer tissues than in noncancerous ones. Consistently, Western blots of pooled proteins extracted from different patients (randomly chosen) also showed an increased IL-22R1 expression in cancerous samples compared with noncancerous tissue counterparts (data not shown). As IL-22R1 has a rather restricted epithelial tissue distribution [8-10], we further examined IL-22R1 overexpression in the two predominant NSCLC histological phenotypes in our large patient cohort: squamous cell carcinoma (SCC; $n=36$ ) and adenocarcinoma (ADC; $n=45$ ). Most commonly, SCCs arise from the pseudo-stratified IL-22R-bearing bronchial epithelial cells of the proximal airways and are strongly associated with smoking, whereas ADCs often have glandular histology and arise in more distal airways [11]. Remarkably, IL-22R1 upregulation was marked in SCC (paired cancerous/ 
noncancerous tissue comparisons, mean $\pm \mathrm{sE} 4.5 \pm 0.8$ fold increase; $\mathrm{p}<0.0001$, Wilcoxon signed-rank test) but not in ADC samples (paired cancerous/noncancerous tissue comparisons, mean $\pm \mathrm{SE} 2.1 \pm 0.4$ fold-increase; $\mathrm{p}=0.153$ ). Also, IL-22R1 upregulation was found to be more than three-fold higher in SCC than in ADC

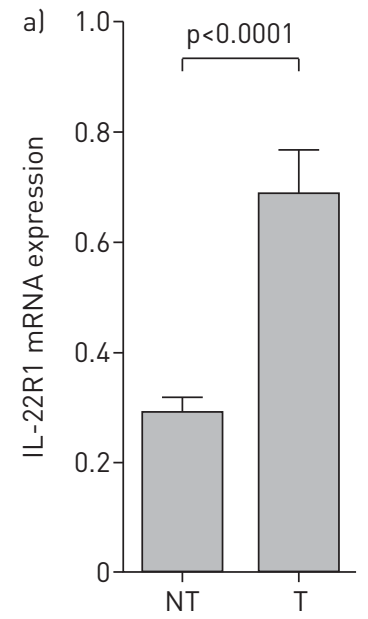

c)

$\operatorname{HR}(95 \% \mathrm{Cl})$

GSE13213 1.14 (0.99-1.32)

GSE31210 1.47 (0.94-2.32)

GSE11117 1.32 (0.90-1.94)

GSE3141 $1.30(0.85-1.97)$

GSE14814 1.05 (0.31-3.55)

GSE4573 $1.98(0.75-5.27)$

GSE17710 $1.25(0.80-1.96)$

Jacob-00182-UM 1.40 (0.82-2.41)

Jacob-00182-CANDF 0.96 (0.26-3.59)

Jacob-00182-HLM 1.47 (0.64-3.36)

Jacob-00182-MSK 1.89 (0.57-6.31)

GSE5846 $1.02(0.41-2.54)$

GSE11969 $2.11(0.46-9.60)$

GSE26939 1.55 (1.19-2.01)

TCGA, ADC 2.36 (1.34-4.14)

TCGA, SCC 0.99 (0.68-1.44)

GSE30219 $1.13(0.95-1.33)$

GSE37745 1.34 (0.82-2.20)

GSE19188 $1.26(0.77-2.06)$

GSE41271 1.24 (1.01-1.53)

GSE50081 1.25 (0.92-1.71)

KM Plotter database 1.37 (1.21-1.56)

CAArray (NCI Lung) 1.37 (1.02-1.84)

GSE29013 2.64 (1.04-6.73)

SurvExpress database 1.20 (1.00-1.44)

Present study 2.33 (1.10-4.92)

Combined 1.27 (1.20-1.35)

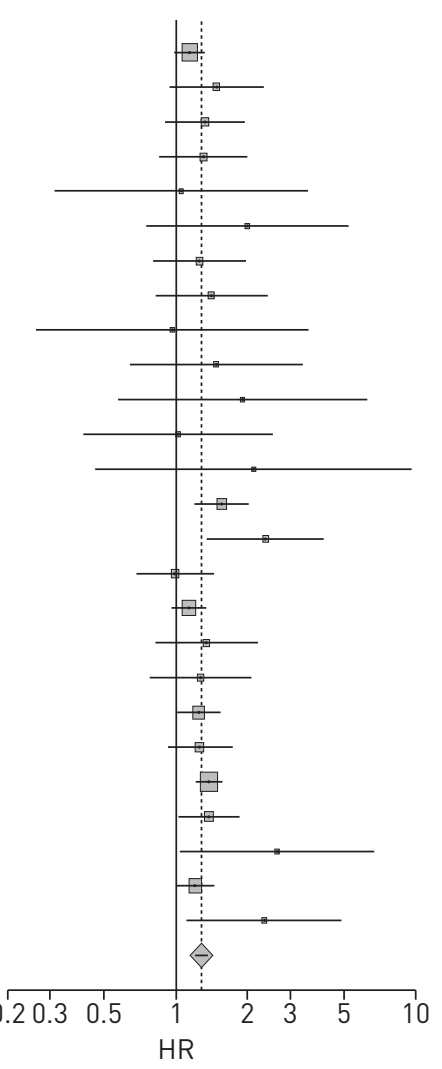

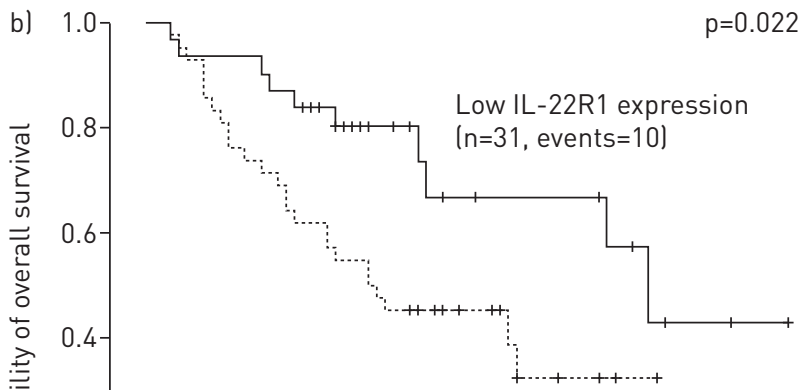

High IL-22R1 expression ( $n=42$, events $=25$ )
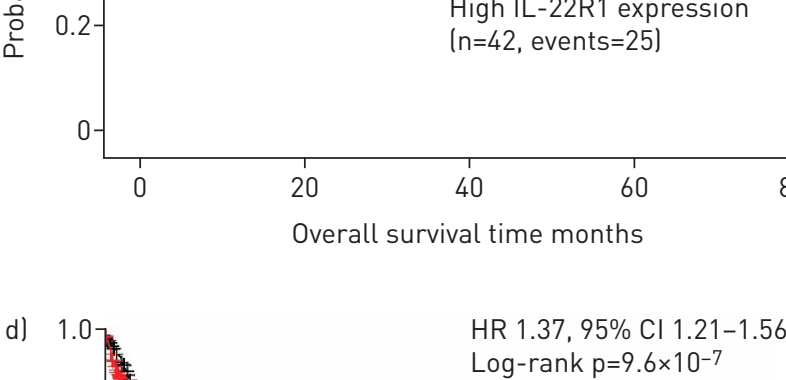

Log-rank $p=9.6 \times 10^{-7}$

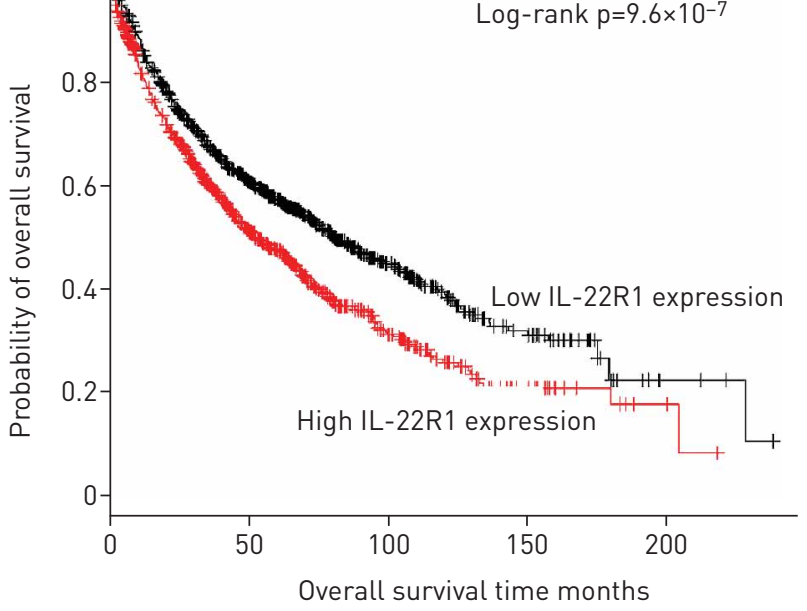

FIGURE 1 High interleukin-22 receptor subunit 1 (IL-22R1) expression is associated with primary nonsmall cell lung cancer (NSCLC) and decreases overall survival. IL-22R1 RNA expression was detected by quantitative reverse transcriptase PCR and normalised to reference genes ( $\beta$-actin and Hprt1) for 81 surgical patients with a histological diagnosis of primary NSCLC. a) IL-22R1 mRNA expression in tumour (T) tissues and nontumour (NT) tissue counterparts was assessed in paired tissues obtained from NSCLC patients. Data are shown as mean \pm SEM. The p-value was calculated by Wilcoxon signed-rank test. b) Kaplan-Meier plots of overall survival in 73 NSCLC patients according to IL-22R1 gene expression levels in tumour. The optimal cut-off point to dichotomise patients into low IL-22R1 expression and high IL-22R1 expression was established by the X-tile algorithm (cut-off value=0.297, 42nd percentile of IL-22R1 expression in tumours). The p-value was calculated by the log-rank test. c) Forest plot of the hazard ratio (HR) for IL-22R1 expression and overall survival by cohort, based on data obtained by the PrognoScan, PROGgeneV2, Kaplan-Meier (KM) Plotter and SurvExpress platforms. d) Prognostic value of IL-22R1 expression in 1926 NSCLC patients through the Kaplan-Meier Plotter database. IL-22R1 tumour expression was dichotomised into high versus low according to median expression. 
histotype ( $\mathrm{p}=0.005$, Mann-Whitney U-test). IL-22R1 mRNA expression in lung tumours was independent of the smoking status $(\mathrm{p}=0.944$ for IL-22R1 mRNA expression comparison between never-smokers and current/former smokers, Mann-Whitney U-test). However, among smokers, IL-22R1 expression was significantly upregulated (3.6 times) in tumours of heavy smokers ( $>49$ pack-years) compared with more moderate smokers $(\mathrm{p}<0.001$, Mann-Whitney U-test).

We further evaluated, for the first time, the prognostic value of IL-22R1 expression in primary lung cancer. For that purpose, patient overall survival rates were calculated by the Kaplan-Meier method and compared using the log-rank test. The optimal cut-off point to dichotomise patients into IL-22R1 low expression and IL-22R1 high expression was established by the X-tile algorithm [12]. The analyses were carried out through SPSS Statistics (version 17.0) software (SPSS Inc., Chicago, IL, USA). Among the 73 patients with NSCLC and available follow-up data, Kaplan-Meier survival analysis indicated that NSCLC patients harbouring tumours with high expression of IL-22R1 present a significantly inferior overall survival outcome compared with those with tumours with low IL-22R1 expression ( $p=0.022$ ) (figure 1b). The association of IL-22R1 with unfavourable survival was confirmed by multivariate Cox regression, adjusting for important clinicopathological characteristics including histotypes and stage (hazard ratio (HR) 2.8, 95\% CI 1.2-6.5; $\mathrm{p}=0.014$ ). To further validate the potential prognostic significance of our findings, we interrogated publicly available platforms of expression analysis, including Kaplan-Meier Plotter [13], PrognoScan [14], PROGgeneV2 [15] and SurvExpress [16]. Hazard ratios for overall survival with corresponding 95\% confidence intervals from 26 studies/datasets were charted in a forest plot (performed with StatsDirect software 3.0; StatsDirect Ltd, Altrincham, UK) (figure 1c). IL-22R1 expression was found to be robustly associated with a lower overall survival rate (pooled HR 1.3, 95\% CI 1.2-1.4; $\mathrm{p}<0.0001)$. Finally, we investigated the prognostic value of IL-22R1 expression in NSCLC patients through the Kaplan-Meier Plotter database (probe \#220056_at), which contains updated gene expression data and survival information from a total of 1926 NSCLC patients. High expression of IL-22R1 mRNA was found to be correlated with a poor overall survival (HR 1.4, 95\% CI 1.2-1.6; p<0.0001) (figure 1d). Multivariate analysis adjusted for stage and histotype also confirmed the independent nature of deriving prognostic information from IL-22R1 levels (HR 1.5, 95\% CI 1.2-1.9; p=0.0011).

Overall, by using two independent datasets, our study reveals that the high expression profile of IL-22R1 in NSCLC is an independent indicator of poor overall survival and supports the contribution of the IL-22/ IL-22R pathway in lung cancer development. Further studies are now required to examine whether IL-22R could represent an attractive therapeutic target for NSCLC.

0 @ERSpublications

High expression of IL-22R1 in nonsmall cell lung cancer is an independent indicator of poor overall survival http://ow.ly/WZxKh

Antoine Guillon ${ }^{1,2,3}$, Fabien Gueugnon ${ }^{1,2}$, Konstantinos Mavridis $^{4}$, Emilie Dalloneau ${ }^{1,2}$, Youenn Jouan ${ }^{1,2,3}$, Patrice Diot $^{1,2,5}$, Nathalie Heuzé-Vourc'h ${ }^{1,2}$, Yves Courty ${ }^{1,2}$ and Mustapha Si-Tahar ${ }^{1,2}$

${ }^{1}$ INSERM, Centre d'Etude des Pathologies Respiratoires, U1100, Tours, France. ${ }^{2}$ Université François Rabelais de Tours, Tours, France. ${ }^{3} \mathrm{CHRU}$ de Tours, Service de Réanimation Polyvalente, Tours, France. ${ }^{4}$ Dept of Biochemistry and Molecular Biology, University of Athens, Panepistimiopolis, Athens, Greece. ${ }^{5} \mathrm{CHRU}$ de Tours, Service de Pneumologie, Tours, France.

Correspondence: Mustapha Si-Tahar, Centre d'Etude des Pathologies Respiratoires, INSERM U1100, Faculté de Médecine, 10 boulevard Tonnellé, 37032 Tours Cedex, France. E-mail: si-tahar@univ-tours.fr

Received: Sept 222015 | Accepted after revision: Dec 15 2015 | First published online: Feb 042016

Support statement: Antoine Guillon was funded by an Inserm "poste d'accueil". This funding agency had no role in study design, the collection and analysis of data, the decision to publish or the preparation of the manuscript. There is no other source of support to declare.

Conflict of interest: None declared.

\section{References}

1 Chew V, Toh HC, Abastado JP. Immune microenvironment in tumor progression: characteristics and challenges for therapy. J Oncol 2012; 2012: 608406.

2 Lim C, Savan R. The role of the IL-22/IL-22R1 axis in cancer. Cytokine Growth Factor Rev 2014; 25: 257-271.

3 Sabat R, Ouyang W, Wolk K. Therapeutic opportunities of the IL-22-IL-22R1 system. Nat Rev Drug Discov 2014; 13: 21-38.

4 Wolk K, Kunz S, Witte E, et al. IL-22 increases the innate immunity of tissues. Immunity 2004; 21: 241-254.

5 Kobold S, Völk S, Clauditz T, et al. Interleukin-22 is frequently expressed in small- and large-cell lung cancer and promotes growth in chemotherapy-resistant cancer cells. J Thorac Oncol 2013; 8: 1032-1042.

6 Baird AM, Gray SG, O'Byrne KJ. IL-20 is epigenetically regulated in NSCLC and down regulates the expression of VEGF. Eur J Cancer 2011; 47: 1908-1918. 
7 Zhang W, Chen Y, Wei H, et al. Antiapoptotic activity of autocrine interleukin-22 and therapeutic effects of interleukin-22-small interfering RNA on human lung cancer xenografts. Clin Cancer Res 2008; 14: 6432-6439.

8 Dugger DT, Gerriets JE, Miller LA. Attenuated airway epithelial cell interleukin-22R1 expression in the infant nonhuman primate lung. Am J Respir Cell Mol Biol 2015; 53: 761-768.

9 Guillon A, Jouan Y, Brea D, et al. Neutrophil proteases alter the interleukin-22-receptor-dependent lung antimicrobial defence. Eur Respir J 2015; 46: 771-782.

10 Sonnenberg GF, Fouser LA, Artis D. Border patrol: regulation of immunity, inflammation and tissue homeostasis at barrier surfaces by IL-22. Nat Immunol 2011; 12: 383-390.

11 Chen Z, Fillmore CM, Hammerman PS, et al. Non-small-cell lung cancers: a heterogeneous set of diseases. Nat Rev Cancer 2014; 14: 535-546.

12 Camp RL, Dolled-Filhart M, Rimm DL. X-tile: a new bio-informatics tool for biomarker assessment and outcomebased cut-point optimization. Clin Cancer Res 2004; 10: 7252-7259.

13 Győrffy B, Surowiak P, Budczies J, et al. Online survival analysis software to assess the prognostic value of biomarkers using transcriptomic data in non-small-cell lung cancer. PLoS One 2013; 8: e82241.

14 Mizuno H, Kitada K, Nakai K, et al. PrognoScan: a new database for meta-analysis of the prognostic value of genes. BMC Med Genomics 2009; 2: 18.

15 Goswami CP, Nakshatri H. PROGgeneV2: enhancements on the existing database. BMC Cancer 2014; 14: 970.

16 Aguirre-Gamboa R, Gomez-Rueda H, Martínez-Ledesma E, et al. SurvExpress: an online biomarker validation tool and database for cancer gene expression data using survival analysis. PLoS One 2013; 8: e74250.

\title{
Idelalisib-related pneumonitis
}

\author{
To the Editor:
}

Idelalisib (Zydelig; Gilead Sciences Inc., Foster City, CA, USA) has recently been approved in combination with rituximab for the treatment of refractory chronic lymphocytic leukaemia (CLL) in patients who have received at least one prior therapy, as a first-line treatment in the presence of a 17p deletion or TP53 mutation in patients unsuitable for chemoimmunotherapy, or as a monotherapy in follicular lymphoma refractory to two prior lines of treatment. Idelalisib specifically inhibits phosphatidylinositol 3-kinase $\delta$ (PI3K $\delta$ ), which is part of the B-cell receptor signalling pathway, the activity of which is increased in B-cell malignancies, and which promotes cellular proliferation and survival [1-4]. Here, we report on five patients who were diagnosed with pneumonitis related to idelalisib treatment.

The patients were three men and two women with a median age of 72 years (interquartile range 68-79 years). None had a history of respiratory disease or environmental exposure and none was a current smoker (one had a 7- and another, a 10-pack-year history of smoking). The patients had received idelalisib for a mean time of 7.8 months (range 2-16 years). Four of them were being treated for refractory CLL whereas one had progressive lymphoplasmacytic lymphoma in the setting of Waldenström disease. All patients were receiving idelalisib at a dose of $150 \mathrm{mg}$ twice daily, except for one patient who had been receiving $100 \mathrm{mg}$ twice daily for 14 months (the initial dosing of $150 \mathrm{mg}$ twice daily had to be decreased 2 months after introduction for severe hypertriglyceridaemia). The mean number of previous lines of therapy was 3.8 (range 1-5). For three patients with CLL, idelalisib was initially administered with ofatumumab. At the time of pneumonitis, ofatumumab had been stopped 4-12 months previously. The fourth patient with CLL was receiving rituximab in combination with idelalisib. The patient with Waldenström disease received idelalisib alone. At the time of pneumonitis, three patients had controlled haematological disease, whereas two patients had progressive disease.

All patients complained of cough, dyspnoea and fever. Four had progression of their clinical symptoms over 3-9 weeks, whereas one had progression over 5 days. Four patients had crackles on physical examination and one had a normal examination. One patient had a severe skin reaction attributed to idelalisib toxicity. One patient had diarrhoea.

A lung computed tomography (CT) scan showed diffuse ground-glass opacities $(n=3)$, consolidations $(\mathrm{n}=2)$, diffuse micronodules $(\mathrm{n}=1)$ and pleural effusions $(\mathrm{n}=2)$ (figure 1$)$.

All patients except one required oxygen supplementation and admission to an intensive care unit. Two of these patients required mechanical ventilation. Three of the patients had a bronchoalveolar lavage (BAL). The mean \pm SD total cell count was $583 \pm 380$ per $\mu \mathrm{L}$. Lymphocytic alveolitis was found in two patients (mean lymphocyte proportion $71 \pm 5 \%$ ), whereas one patient had neutrophilic alveolitis (77\% neutrophils, $1 \%$ lymphocytes). An extensive search for pathogens, including respiratory viruses, bacteria and fungi, was 